\title{
564
}

XXXVII. Durchsichtiges Platin auf Crownglas.

(In Luft reflectirtes Lirht.)



Man sieht, wie im Allgemeinen die beobachteten und berechneten Werthe übereinstimınen.

Auf den Einflufs, welchen Dicke der Metallschicht auf Phasenunterschied und Azimuth hat, werde ich später noch zurückkommen.

Berlin, im Mai 1866.

IV. Ueber die Ausdehnung starrer Körper durch die Wärme; von Hrn. Fizeau.

(Comp. rend. T. LXII, p. 1101 et 1133.)

Erster Theil.

Bereits batte ich die Ehre der Akademie meine ersten Versuche uiber die Ausdebnung starrer, besonders krystallisirter Körper durch die Wärne, vorzulegen ${ }^{1}$ ). Die Natur der am Bergkrystall, Diamant, Kupferoxydul und an mehren anderen Substanzen beobachteten Erscheinungen, die Sauberkeit der durch die Methode der Wellenlängen

1) Vergl. Ann. Bd. CXXIII, S. 515; Bd. CXXVI, S. 611. 
erhaltenen Resultate, endlich die Leichtigkeit und Sicherheit der Beobachtungen, haben mich veranlafst, diese Untersuchungen foltzusetzen und, wie ich hoffe, zu erweitern. Heute gebe ich den ersten Theil meiner neuen Studien, in welchem ich einige allgemeine, mehr geometrische als physikalische Sätze in Betreff der Ausdehnungsphänomeue krystallisirter Substanzen untersuchen werde.

Betrachtet man zuvörderst die durch Wärme bewirkten Volumsveränderungen bei amorphen und homogenen Körpern, welche in allen Punkten und nach allen Richtungen einerlei Molecular - Constitution besitzen, so müssen die Ausdehnungsphänomene dieselbe Einfachheit und Einförmigkeit darbieten, welche man in Betreff anderer physikalischer Eigenschaften z. B. der tönenden Elasticităt, der Wärmeleitungsfähigkeit, der Wirkung des polarisirten Lichtes usw. an ihnen beobachtet hat.

Bei krystallisirten Körpern dagegen, welche die mannigfaltigsten und scheinbar oft die unregelmäfsigsten polyëdrischen Formen darbieten, obwohl sie immer einer gewissen Anordnung und wohl bestimmten Symmetriegeselzen in Bezug auf die Spaltbarkeit unterworfen sind, ist man genöthigt, eine ganz andere innere Structur anzunehmen, die ohne Zweifel zwar in allen Theilen eines Krystalls homogen, aber je nach der betrachteten Richtung verschieden ist. Diese Besonderheiten der inneren Structur müssen sich unfehlbar durch ungleiche Ausdehnungen nach verschiedcnen Richtungen kund gebeu, so gut, wie durch entsprechende Modificationen in den eben erwähnten physikalischen Eigenschaften.

Diese Modificationen der physikalischen Eigenschaften, je nach den verschiedeuen Richtungen, befolgen indefs eine gewisse Ordnung in Bezug auf die Lage der Krystallflächen und die allgemeine Symmetrie der Krystalle; und diese Ordnung tritt besonders hervor, wenn man gewisse feste Richtungen, die Fresnel bei der Theorie der Doppelbrechung angewandt hat, in Betracht zieht. Ich meine die drei rechtwinkligen Richtungen, die man Elasticitäts. 
axen nennt, und um welche sich nicht allein alle optischen Erscheinungen der Doppelbrechung ein- und zweiaxiger Krystalle in vollkommener Ordnung gruppiren, sondern auch die hauptsächlichsten Symmetriegesetze der verschiedenen Krystallsysteme, die Beobachtungen über die tönenden Schwingungen krystallisirter Platten, die Entdeckungen S en armont's über die Fortpflanzung der Wärme in Krystallen und endlich die Beobachtungen Fresnel's und Mitscherlich's über die ungleiche Ausdehnung mehrer krystallisirter Körper.

Messungen der Ausdehnung an einer grofsen Auzahl krystallisirter Körper, die ich weiterhin in dieser Arbeit beibringen werde, stimmen mit diesen, an sich schon sebr sicheren Betrachtungen überein, um festzustellen, dafs die hauptsächlichsten Phänomene der Ausdehnung der Krystalle von der Lage der Elasticitätsaxen abhängen, in demselben Grade, wie die übrigen physikalischen Eigenschaften. Demgemäfs mufs man annehmen, dafs es in einem krystallisirten Körper drei unter sich rechtwinklige Richtungen giebt nach welchen sich drei Haupt-Ausdehnungen $\alpha, \alpha^{\prime}, c^{\prime \prime}$ äulsern; die eine dieser Richtungen repräsentirt die grölste Linear-Ausdehnung der Substanz, die zweite die kleinste und die dritte eine mittlere. Die combinirten Effecte dieser drei Ausdehnungen sind es, welcbe zu den mannigfachen Ausdehnungen in anderen Richtungen Anlafs geben.

Es ist überdiefs zu bemerken, dals diese complexen Ausdehnungen nothwendig begleitet sind von kleinen Veränderungen in den Winkeln zwischen den Krystallfächen, die jedoch immer eben bleiben, und dafs diese Winkel sich, iunerhalb der Beobachtungsgränzen, um so kleine Gröfsen verändern, dal's man dieselben als ganz zu vernachlässigen betrachten kann.

Aus dem Vorhergehenden folgt, dafs wenn man die Volumsveränderung oder die cubische Ausdehnung einer Substanz mittelst direct beobachteter linearer Ausdehnungen bestimmen will, man im Allgemeinen drei gesonderte Bestimmungen in den drei bezeichneten Richtungen machen 
mufs. Die Summe der drei Werthe giebt, unter Vernachlässigung der Grölsen zweitcr Ordnung, den Werth der cubischen Ausdehnung. Einige Substanzen sind auf diese Weise studirt worden; allein die zu solchen Beobachtungen erforderliche Zeit und die Schwierigkeit, die oft sehr kleinen Krystalle zu schneiden, mufsten nothwendig diese Art von Bestimmungen sehr beschränken. Ich bin daher veranlafst worden, zu untersuchen, ob es nicht einige besondere Richtungen gäbe, die geeignet wären, die Beobachtungen zu erleichtern, und ich glaube wirklich dahin gelangt zu seyn, eine durch Einfachheit und Allgemeinheit merkwürdige Beziehung entdeckt zu baben, die, wie ich hoffe, einen bedeutenden Fortschritt im Studium der Ausdehnungen darstellt, indem sie die Beobachtungen in unerwarteter Weise erleichtert.

Ich suchte zunächst einen geeigneten Ausdruck für die Ausdehnung, die ein zu den drei Krystalltypen gehöriger Krystall nach irgend einer gegebenen Richtung hin erleidet, welche die Winkel $\delta, \delta^{\prime}, \delta^{\prime \prime}$ mit den drei rechtwinkligen Elasticitätsaxen bildet, nach welchen die drei Haupt-Ausdehnungen $\alpha, \alpha^{\prime}, \alpha^{\prime \prime}$ stattfinden.

Diese Ausdehnung kann in Function der drei Winkel und dreier Coëfficienten ausgedrückt werden, wenn man folgenden Gang einschlägt.

Durch eine der Axen ( $\alpha$ ) und die gegebene Richtung denke man sich eine Ebene gelegt, welche die Ebene der beiden anderen Axen $\left(\alpha^{\prime}\right),\left(\alpha^{\prime \prime}\right)$ nach einer geraden schneide, die in dieser letzten Ebene mit einer dieser Axen $\left(\alpha^{\prime}\right)$ den Winkel $\beta$, und mit der anderen $\left(\alpha^{\prime \prime}\right)$ den Winkel $90^{\circ}-\beta$ macht. Der Winkel $\beta$ ergiebt sich mittelst eines sphärischen Dreiecks aus den gegebenen Winkeln $\delta$ und $\delta^{\prime}$.

Nun suche man in der Ebene $\left(\alpha^{\prime}\right)\left(\alpha^{\prime \prime}\right)$ den Werth der Ausdebnung nach $\beta$, welcher sich ergeben mufs aus den beiden unter sich rechtwinkligen, in dieser Ebene liegenden Ausdehnungen $\alpha^{\prime}$ und $\alpha^{\prime \prime}$. Einige Hülfsdreiecke führen zu dem Ausdruck:

$$
\alpha=\alpha^{\prime} \sin ^{2} \beta+\alpha^{\prime \prime} \cos ^{2} \beta \text {. . . . }
$$


Das ist in einer Ebene der Ausdruck für die Ausdehnung nach irgend einer Richtung $\beta$ unter dem Eiuflufs zweier unter sich rechtwinkliger und immer als sehr klein vorausgesetzter Ausdehnungen. Allein diese Richtung $\beta$, für welche man so die Ausdehnung $d$ findet, liegt auch in der ursprünglich betrachteten Ebene $(\alpha) \beta$ und ist rechtwinklig auf der Axe $(\alpha)$; überdiefs befindet sich in dieser Ebene die gegebene Richtung, welche mit der Axe $(\alpha)$ den gegebenen Winkel $\delta$, und mit der neuen Richtung $\beta$ den Winkel $90^{\circ}-\delta$ bildet. Dieselbe Formel wie in dem vorhergehenden Fall kann angewandt werden, und man hat für die gesuchte Ausdehnung $D$ nach der gegebenen Richtung:

$$
D=d \sin ^{2} \delta+\alpha \cos ^{2} \delta \quad . \quad \text {. }
$$

Substituirt man in diesem Ausdruck den Werth von $d(1)$ und drückt $\beta$ in Function von $\delta$ und $\delta^{\prime}$ aus durch die Relation $\cos ^{2} \beta=\frac{\cos ^{2} \delta^{\prime}}{\sin ^{2} \delta^{\prime}}$, so gelangt man uach einigen Transformationen zu der Endformel:

$$
D=\alpha \cos ^{2} \delta+\alpha^{\prime} \cos ^{2} \delta^{\prime}+\alpha^{\prime \prime} \cos ^{2} \delta^{\prime \prime} \quad \text {. (3). }
$$

Das ist der Ausdruck, welcher für irgend einen Krystall und für einen kleinen Temperaturanwuchs giebt: den Anwuobs der Längen-Einheit nach irgend einer Richtung, die mit den drei rechtwinkligen Elasticiätsaxen des Mittels die Winkel $\delta, \delta^{\prime}, \delta^{\prime \prime}$ macht, und zwar in Function der diesen drei Axen entsprechenden Haupt-Amsdehnungscoëlficienten $\alpha, \alpha^{\prime}, \alpha^{\prime \prime}$.

Bekantlich wird die cubische Ausdehnung der Volum. Einheit für einen kleinen Temperatur-Anwuchs im Allgemeinen geben durch die Summe $\alpha+\alpha^{\prime}+\alpha^{\prime \prime}$ der drei Haupt-Ausdehnungen, die irgend welche, positiv oder negativ́ (Ausdehnungen oder Zusammenziehungen) seyn können, sobald sie nur in der Gröfsen-Ordnung bleiben, welche die Erfahrang bei den bekaunten Körpern als geeigvet für diese Grörsen befuarden hat.

Für die Krystalle des regelmäfsigen Systems (den Würrfel) sind die drei linearen Ausdehmnngen gleich und die 
cubische Ausdehnung wird $3 \alpha$. Man hat also die einzige lineare Ausdehnung

$$
L=\frac{a^{\text {eub. }}}{3}
$$

Für die Krystalle der rings um eine der Elasticitätsaxen symmetrischen Systeme (gerades quadratisches Prisma, hexagonales Prisma und Rhomboëder) sind die beiden auf dieser Axe winkelrechten Ausdehnungen $\alpha^{\prime}, \alpha^{\prime \prime}$ unter sich gleich; die dritte $\alpha$, längs der Axe, ist verschieden. Die cubische Ausdehnung wird dann $\alpha+2 \alpha^{\prime}$, wo $\alpha^{\prime}$ die oberflächliche Ausdehnung einer auf der Axe winkelrechten Ebene vorstellt; die mittlere, lineare Ausdehnung wird

$$
L=\frac{\alpha+2 \alpha^{\prime}}{3} \text {. }
$$

Bei den Systemen des geraden und des schiefen rhombischen Prismas, sowie des doppelt schiefen Prismas, sind die drei Ausdehnungen im Allgemeinen ungleich, und die cubische Ausdehnuug ist $\alpha+\alpha^{\prime}+\alpha^{\prime \prime}$; alsdann ist die mittlere lineare Ausdehnung

$$
L=\frac{\alpha+\alpha^{\prime}+\alpha^{\prime \prime}}{3}
$$

Dieser Ausdruck scbliefst die beiden vorhergebenden als besondere Fälle ein.

Wie man sieht kann man in jedem der drei vorhergebendeu Fälle zur Kenntnifs der Volumveränderung oder der cubischen Ausdehnung gelangen, wenn man entweder einen, oder zwei oder drei besondere lineare Ausdehnungscoëfficienten bestimmt. Allein man kann dahin auch auf einem einfacheren und sehr allgemeinen Wege gelangen, der sich aus dem vorhin für die Ausdehnung nach irgend einer Richtung gefundenen Ausdruck ergiebt.

In der That nimmt man wieder die Gleichung:

$$
D=\alpha \cos ^{2} \delta+\alpha^{\prime} \cos ^{2} \delta^{\prime}+\alpha^{\prime \prime} \cos ^{2} \delta^{\prime \prime} . .
$$

und bemerkt, dafs die drei Winkel $\delta, \delta^{\prime \prime}, \delta^{\prime \prime \prime}$ mit einander verknüpft sind durch die bekannte Relation:

$$
\cos ^{2} \delta+\cos ^{2} \delta^{\prime}+\cos ^{2} \delta^{\prime \prime}=1 \quad \text {. }
$$

so fimdet unan, dafis für den Fall, wo die betrachtebe Rich- 
tung gleich geneigt ist gegen die drei Axen, d. h. für $\delta=\delta^{\prime}=\delta^{\prime \prime}$, die Gleichung (4) giebt:

$$
\cos ^{2} \delta=\frac{1}{3} ; \quad \delta=54^{\circ} 44^{\prime} ;
$$

allein alsdann wird die Gleichung (3)

$$
D=\frac{\alpha+\alpha^{\prime}+\alpha^{\prime \prime}}{3}
$$

was genau die oben mit $L$ bezeichuete Gröfse ist, d. h. die mittlere Ausdehnung des Krystalls im allgemeinsten Fall.

Man sieht also, dafs für eine gegen die drei Elasticitätsaxen gleich geneigte Richtung von $54^{\circ} 44^{\prime}$ die Ausdehnung genau der mittleren linearen Richtung gleich ist, welches Krystallsystem auch die Substanz habe und welche Werthe, positive oder negative, die drei Haupt Ausdehnungscoëfficienten des Krystalls auch besitzen.

Man wird auch bemerken, dafs diese Richtung die der Diagonale des Würfels in Bezug auf seine Axen ist, und folglich winkelrecht auf der Fläche des regulären Octaë-. ders, welches daraus entspringt. Ueberdiefs ist klar, dafs dasselbe Raisonnement für andere ähnliche Richtungen in dem Krystall gilt, d. h. dafs es sich wiederholt für die acht Ecken, welche von den drei Ebenen der Elasticitätsaxen gebildet werden.

Man kann sich im Innern eines jeglichen Krystalls ein reguläres Octaëder so gestellt denken, dafs seine Flächen gegen die drei Elasticitätsaxen gleich geneigt seyen; dann wird man winkelrecht auf den Flächen dieses Octaëders die mittlere Ausdehnung dieses Krystalles haben.

Im Fall die Richtung der Elasticitätsaxen unbekannt wäre, kann man sich im Innern des Krystalls einen Würfel irgendwie gelagert denken und man wird dann die cubische Ausdehnung erhalten, wenn man die Summe der drei linearen Ausdehnungen nimmt, die in den drei auf den Flächen des Wtirfels winkelrechten Richtungen gemessen worden sind. Dieser Satz ergiebt sich leicht aus den Relationen (3) und (4).

Die eben aufgestellten Principien erlauben, wie man 
sieht, die Ausdehnungen unter den scheinbar verwickeltsten Umständen zu untersuchen und die Volumveränderungev verschiedener Körper dadurch mit Sicherbeit zu erhalten, dafs man die linearen Ausdehnungen nach gewissen wohl bestimmten Richtungen milst, was sich leicht bewerkstelligen · lälst.

Ich will nun einige Details über dic experimentelle Einrichtung angeben, bei welcher ich stehen geblieben bin, nachdem ich die verschiedenen Umstände, die bei den numerischen Bestimmungen zu merklichen Fehlern Anlafs geben könnten, sorgfältig studirt hatte; dann werde ich die Resultate der Beobachtungen beibringen, die icb an einer grofsen Anzahl amorpher oder krystallisirter, ihrer WärmeAusdehnung nach meistens ganz unbekannter Substanzen angestellt habe.

\section{Zweiter Theil.}

Ich werde die Principien der früher beschriebenen Beobachtungsmethode ${ }^{1}$ ) in einigen Worten wiederholen, um die seitdem zur Erhöhung der Genauigkeit der neuen Bestimmungen getroffenen Einrichtungen deutlicher auseinandersetzen zu können.

Man giebt dem zu untersuchenden Stück des Krystalls oder der Substanz zwei einander parallele und polirte Flächen, und legt es, so vorbereitet, frei auf den Teller eines kleinen metallenen Dreifufses, dessen Füfse aus drei Schrauben bestehen, die durch den Teller gehen und oben in stumpfen Spitzen endigen. Auf diesen drei Spitzen und der Substanz sehr nahe ruht eine polirte Glastafel, deren Abstand von der oberen Fläche der Substanz durch die Bewegung der drei Schrauben so regulirt werden kann dafs das Phänomen der New ton'schen Farbenringe zum Vorschein kommt.

Für die in Rede stehenden Beobachtungen erzeugt man die Ringe oder Fransen mit dem Lichte einer durch Koch-

1) Compt. rend., Mai, 1864 und Ann. de chim. et de phys. Sér. IV, T. II (Ann. Bd. CXXIII S. 515). 
salz gelb gefärbten Flamme, und mittelst einer Reflexion und einigen einfachen optischen Vorrichtungen kann man die auf der Oberfläche der Substanz erzeugten Ringe oder Fransen in distans unter normaler Incidenz beobachten. Die Lage der Fransen und die eintretenden Verschiebungen derselben werden bestimmt in Bezug auf feste Punkte; die regelunäfsig in die Glastafel eingegraben sind. Es sind wahrhafte Visirpunkte, die gleichzeitig mit den Fransen im Fernrohr sichtbar sind.

Diesen kleinen, sehr einfachen Apparat stellt man in die Mitte eines kleinen Ofens von doppelten Wänden aus sehr starkem Kupfer, der durch zwei recht constante Lampen erhitzt werden kann. Zweckmärsige, durch Glasplatten verschlossene Oeffinungen und zwei Thermometer mit kurzen Stielen und grofsen die Substanz und den Dreifufs fast berührenden Behäktern erlauben in jedem Augenblick die Lage der Fransen und die entsprechenden Temperaturen zu beobachten.

Ohne weiter in die Details einiger Hülfsvorrichtungen einzugehen, lälst sich sagen, dafs der Apparat in Ganzen wenig voluminös, leicht zu handbaben, und merkwürdig regelmäfsig in seinen Functionen ist ${ }^{1}$ ).

Der bei den früheren Versuchen von Stahl verfertigte Dreifufs ist jetzt ${ }^{2}$ ) von geschmolzenem Platin mit 0,1 Iridium, nach dem Verfahren des Hrn. Henri S a in te-Claire Deville. Die Vorzüge des Platins in diesem Falle bestehen in seiner gröfseren Dichtigkeit, welche einen festeren Stand bedingt, in seiner Unveränderlichk eit, seiner Ausdehnungsweise, welche weniger als die des Stahls mit der Temperatur variirt, und endlich seiner Feuerbeständigkeit, die verstattete, den Dreifuls in Laboratorium der École Normale mehre Standen hindurch der Rothgluth ohne Schaden auszusetzen. Diese Operation schien nothwendig, um

1) Dieser Apparat ist won Hrn. Soleil construirt, dessen wohlbekannte Geschicklichkeit im Schleifen der Krystalle mir aufserdem bei diesen Untersuchungen von grofsem Nutzen war.

2) Von den HH. Brunner verfertigt. 
jede Spur von Härtung zu zerstören, welche kleine Unterschiede in der Ausdehnung der verschiedenen Theile der Schrauben und des Tellers hätte veranlassen können.

Mit besonderer Sorgfalt strebte ich bei diesen Versuchen eine genaue Kenntuifs der Temperaturen zu erlangen; zu dem Ende machte ich eine directe Bestimmung der Fehler in der Theilung der angewandten Thermometer, indem ich dieselben mit einem von Hrn. R e gnault geprüften Normalthermometer verglich. Zu diesem Behufe tauchte ich die drei Instrumente ganz in ein Gefäls, dessen eine senkrechte Wand aus Glas bestand, und das etwa 50 Liter enthielt, welches auf verschiedene Temperaturen erhitzt und vor jeder Ablesung mittelst eines Agitators umgerührt wurde. Nach dieser Vergleichung wurde für jedes der beiden Thermometer eine Correctionstafel entworfen, mittelst deren die Angaben dieser Instrumente eine grofse Genaugkeit erlangten. Die kreisrunde Gestalt der beiden concentrischen Ofenwände, die Dicke und die Leitungsfähigkeit ihres Metalls und vor allem die Sorgfalt, dafs inan keine Beobachtung anders als bei wenig erhöhten und stundenlang ganz stationär gewordenen Temperaturen machte, - alle diese Umstände nebst verschiedenen sehr befriedigenden Verificationen, die aus den Beobachtungen selbst hervorgingen, berechtigen die Beobachtungen für vollkommen zuverlässig zu halten. - Ich schätze die Unsiçherheiten, die bei den höheren Temperaturen am merklichsten seyn müssen, nicht über ein Zehntelgrad.

Es ist gut sich zu erinnern, dafs wenn ein wa studirender Krystall in den Apparat gelegt und die Temperatur erhöht wird, man die Oberfläche des Krystalls durch das Fermrohr ganz mit breiten Fransen bedeckt sieht, welche sich gegen eine gewisse Anzahl schwarzer Punkte, die als Merkzeichen dienen, langsam verschieben. Man weifs auch, dafs, wenn eine ganze Franse vom Centrum aus nach der Peripheric durch einen Visirpunkt geht, der Zwischenraum, worin sich zwischen dem Krystall und der Glasplatte die Fransen bilden, um die Länge einer halben Welle $\frac{1}{2} \lambda_{\text {p }}$ des 
gelben Lichtes d. h. um $\frac{1}{2}\left(0^{\mathrm{mm}}, 0005838\right)$ abgenommen hat, so dafs man immer $n$ Fransen $=\frac{1}{2} n \lambda$ hat. Man braucht also nur die Hälfte der ganzen oder gebrochenen Anzahl verschobener Fransen zu nehmen und sie mit dem Werth von $\lambda$ zu multipliciren, um das Maals des gegenseitigen Abstandes beider Flächen sehr genau in Millimetern zu erhalten ${ }^{1}$ ).

Diefs ist die scheinbare Ausdehnung der Substanz für ihre Dicke $e$ und für die Temperatur-Erhöhung $t^{\prime}-t$. Allein diese scheinbare Ausdehnung $D^{\prime \prime}$ ist offenbar gleich dem Unterschied zwischen der wirklichen Ausdehnung $D$ der Substanz, welche den Abstand zwischen den Flächen zu vermindern strebt, und der Ausdehnung $D^{\prime}$ der Platinschraube, welche, indem sie die Glastafel hebt, diesen $A b$ stand zu vergröfseru sucht. Man hat also $D^{\prime \prime}=D-D^{\prime}$,

1) Ich habe die, übrigens ganz sichere Richtigkeit dieses Principes, sowie den numerischen Werth von $\lambda_{D}$, welcher sich aus den Fraunhofer'schen Beobachtungen der. Gitterphönomene ergiebt, durch folgende Versuche zu controliren gesucht.

Lin kleines Instrument, analog eiver Theilmaschine, aber gebildet aus einer Schraube, bestehend aus zwei Theilen, deren Gang elwa um ts verschieden ist, wurde nach meinen Angaben von Hrn. Duboscq verfertigl; es ist in zwei mit Furchen laufenden Schlitten versehen, einem festen und einen andern, der durch die Differentialschraube ungemein langsam verscboben werden kann. Man bildete die Newton'schen Ringe mit gelbem Lichte zwischen zwei Glastafeln, von denen die cine von dem festen Schlitten und die andere, mit Visirpunkten versehene, von dem beweglichen getragen wird; auf diesem letzteren war überdiefs ein Froment'sches Glasmikrorneter befestigt, dessen Abtheilungen $=0^{\mathrm{mm}}, 05$ mittelst ejnes unbeweglichen, mit Fadenkreuz versehenen Mikroskops beobachtet wurden.

Erste Beobachtung. - Die Glastafeln fast in Contact; als der be-

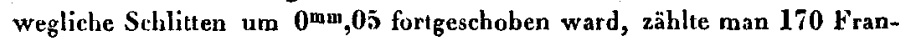
sen durch einen selben Visirpunkt gehend.

Zweite Beobachtung. - Abstand zwischen beiden Glastafeln etwa $7^{\mathrm{mm}}, 2$. Vährend der bewegliche Schlitten $0^{\mathrm{mm}, 05}$ vorrückte, zählte nan dieselbe Anzahl verschobener Fransen, d. h. 170. Das Phänomen berechnet mit der Fraunhofer'schen Vellenlänge $\lambda_{D}=0^{\mathrm{mm}, 0005888}$ hätte $169^{\text {fr, }}, 84$ geben müssen, eine Uebereinstimmung, die man als genügend betrachten mufs, wenn man die ziemlich beschränkte Genaujgbeit dieses Apparats in Erwägung zieht. 
woraus man sieht, dals, wenn $D^{\prime}$ bekannt wäre, man habeu würde $D=D^{\prime \prime}+D^{\prime}$, d. h. die gesuchle Ausdehnung wäre die Summe der Ausdehnung der Platinschrauben und der beobachteten scheinbaren Ausdelnnung des Platin und man erhielte den Ausdehnugscoëlficienten, wenn man diesen Werth durch $e\left(t^{\prime}-t\right)$ dividirte.

Die Methode erfordert also die vorgängige und sehr genaue Bestimmung der Ausdehnung des Metalls, aus welchem der Dreifuls verfertigt ist ${ }^{1}$ ).

Ich will jetzt die Versuche beibringen, die ich machte, um die Constanten der Ausdehnung des neuen platinern Dreifufses, der zu allen ferneren Versuchen dienen sollte, zu bestimmen.

Zwischen der oberen Glastafel und dem polirten Teller des Dreifufses bildete ich Ringe oder Fransen von grolsem Gang-Unterschied. Der für die Mehrzahl der Versuche gewählte Abstand oder die Länge der Schrauben betrag $\left.10^{\mathrm{mm}}, 361\right)$ und für einige $3^{\mathrm{mm}}, 387$, was erlaubte nachzuweisen, dafs die Schrauben sich in ihrer ganzen Länge recht gleichmäfsig ausdehnten.

Der Dreifuls wurde num in die Mitte des Ofens (in welchen zur Austrocknung der Luft einige Stückchen Aetzkali gelegt worden) gestellt und darauf die Lage der Fransen in Bezug auf zehn Visirpunkte bestimmt, erst bei der umgebenden Temperatur, etwa $12^{\circ} \mathrm{C}$. und dann bei imner

1) Man wird bemerken, dals wenn man die Ausdehsung einer Substanz mit grofser Genauigkeit kannte, man daraus die des Metalls des Dreifufses mit ähnlicher Genaugkeit herleiten könnte. Da nun die Bestimmung dieser letzteren Ausdehnung für den Dreifufs eines jeden Apparats besonders gemacht werden mufs, so habe ich gesucht, den Physikern, die sich mil dieserartigen Beobachtungen befassen wollen, die Mühe zu erleichıen. Es bedarf nämlich dazu (womit ich mich jetzt beschäftige) nur der sehr genauen Bestimmung (durch Vervielfäligung der Versuche und Mittelziehung aus mehrea Reihen von Messungen) der beiden Ausdehnungs - Constanten einer wohl charakterisirten Substanz, die jeder Beobachter sich im Zustande der vollkommensten Reinheit und Identität verschaffen kann (Flufsspath z. B.). Es wird dann ein Leichtes, die Ausdehnungs - Constanten des Dreifulses eines jeden neu construirten Apparates in kurzer Zeit und selir sicher zu bestimmen. 
höheren Temperaturen bis $80^{\circ}$ (der Apparat war zuvor oline ibn zu beobachten bis $90^{\circ}$ erhitzt worden). Im Moment jeder Beobachtang las man die beiden Thermometer des Ofens und das äufsere Barometer ab. Wie schon bemerkt, wurden die Beobachtungen nur dann für gut gehalten, wenn die von den Thermometern angezeigte Temperatur und die Lage der Fransen zwei bis drei Stunden hindurch ganz constant geblieben waren (vorausgesetzt, dafs das Barometer keine merkliche Veränderung gezeigt hatte).

Aus einigen vorläufigen Versuchen wufste man, wieviel Fransen für eine gegebene Anzahl von Graden vorüberginge (ungefähr $18^{\text {fr }}, 1$ für $63^{\circ}, 7$ ); die ganze Auzahl war deunnach immer mit Sicherheit bekannt und die Bruchzabl wurde genau erhalten, indeu man die Summe der griofsten und kleinsten Excursionen der Fransen in Bezug auf jeden der zehn Visirpunkte nahm, und aus den zehn also gefundenen Werthen das Mittel nahm.

Allein diese Anzahl verschobener Fransen erfordert eine Berichtigung, hervorgehend aus den Wirkungen der durch die Wärme ausgedehnten Luft und zuweilen aus dem Einflufs einer merklichen Aenderung des Barometerstandes, wïhrend der Zwischenzeit von einem Minimum zu einem Maximum der Temperatur (etwa 8 bis 10 Stunden). Diese Berichtigung wird in Fransen-Anzahl gegeben durch die Formel:

$$
\varphi=+\frac{2 e}{\lambda}\left(m-m^{\prime}\right)
$$

wo $e$ die Länge der Schrauben, $m$ und $m^{\prime}$ die Brechungsindexe der trocknen Luft, hergeleitet aus dem Bi o t- Arago'schen Gesetz ${ }^{1}$ ), für niedrigere und höhere Temperaturen

1) Das Biot-Arago'sche Gesetz über die Veränderungen des Brechungsindexes der Gase mit der Temperatur und dem Druck ist folgendes:

$$
\frac{m^{2}-1}{d}=\frac{m^{\prime 2}-1}{d}
$$

d. I. wenn anch die Dichtigkeit sich durch Aendesungen der Tempe- 
und für entsprechende Drucke. Nennt man $f^{\prime}$ die beobachtete Verschiebung, so hat man für die im Vacuo stattfindende Verschiebung

$$
f=f^{\prime}+\varphi
$$

ratur und des Drucks verändert, bleibt doch die Grörse $\frac{m^{2}-1}{d}$ oder das Brechungsvermögen constant. Diefs Gesetz ist besonders für Druckveränderungen festgestellt worden; für Temperaturveränderungen waren die Beobachtungen weniger zahlreich und weniger sicher; da nun äberdiefs, bei Anwendung auf die obigen Versuche, die mittelst dieses Gesetzes berechnete Correction $\Phi$ ein beträchtlicher Bruch (zuweilen $\frac{1}{10}$ ) der beobachteten Grölse $f^{\prime}$ ist, so war es wichtig die Richtigkeit des Gesetzes durch neue Beobachtungen zu controliren, wenn es auch niemals mangelhaft befunden zu seyn scheint. Diefs habe ich durch die folgenden Versuche zu thun gestrebt; der Erfolg derselben war freilich nicht vollständig, reicht aber doch hin zu der Berechtigung, dieses Gesetz bei den Rechnungen als hinreichend genauen Ausdruck der Phänomene anzuwenden.

Ich nahm einen kleinen Glascylinder von $8^{\mathrm{mm}, 0}$ Höhe, verschlossen durch zwei dünne Glasplatten, die ringsum am Umfang hervorragten. In der Mitte des Cylinders war. eine dünne Glasröhre angelöthet, die gestattete den Cylinder auszupumpen, auszutrocknen und durch Zuschmelzen ihrer Spitze zu verschliefsen. Nachdem dieser kleine luftleere Apparat auf den Teller des Dreifufses gestellt worden, konnte man gleichzeitig die Ringe beobachten, welche sich zwischen dem Teller und der Ebene des oberen Glases sowohl in dem inneren lufteeren Theil, als auch im äufseren Theil rings um den Cylinder und folglich in der Luft gebildet hatten; da der Effect der kleinen Glasplatten derselbe war auf diese beiden Theile von Ringen, so fand swischen besagten Theilen nur vermöge des Contrastes der Luft und des Vacuums eine Verschiedenheit statt. Nun war die Verschiebung der Fransen in der That verschieden in beiden Fällen, und zwar um eine Gröfse, welche der vom Calcäl als Berichtigung unter denselben Umständen von Temperatur und Dicke angegebenen sehr nahe kam, obgleich nicht strenge gleich war. Der Sinn des Unterschiedes deutete darauf, dafs entweder der Index der Luft etwas rascher abnahm als zufolge des Gesetzes, oder dạfs bei der Erhitzung eine merkliche Entwickelung von Vasserdampf in dem leeren Raum stattfand. Diese letztere Ursache schien am wahrscheinlichsten zu seyn und man versicherte sich, dals man wirtlich, ungeachtet der Sorgfalt den Cylinder vor seinem Verschlusse zu trocknen, darin einige Tröpfchen Wasser verdichten konnte, als man ihn erst erhitzte und dann auf seine Oberfläche ein Stück Eis legte. Kurz dieser Versuch zeigle, dals die nach dem erwähnten Gesetz berechnete Correction bis auf 0,1 richtig Poogendarffs Annal. Rd rXXVIII. 
und daraus ergiebt sich der Ausdehnungscoëfficient $\alpha_{\text {s }}$ unmittelbar:

$$
a^{2}=\frac{f}{2 e\left(t^{\prime}-t\right)} .
$$

$t^{\prime}-t$ ist der Unterschicd zwischen den extremen Tem. peraturen, und der Index $i$ entspricht $\frac{1}{2}\left(t^{\prime}+t\right)$ d. h. dem mittleren Grad bei der Beobachtung. Die Erfahrung hat nämlich gezeigt, dafs der Ausdehnungscoëfficient, d. h. die Zunahme der Längen-Einheit für einen Grind im Allgemeinen nicht fest ist für verschiedene Punkte der Thermometerscalc, sondern bei allen bekannten Substanzen merklich wächst mit steigender Temperatur; allein es ist nach den neuen Beobachtungen gleichfalls sicher, dafs dieser Anwuchs des Ausdehnungscoëfticienten beinahe proportional ist dem Temperatur-Ueberschufs, d. h. gleich für alle Grade in der ganzen Thermometerscale (von $10^{\circ}$ bis $85^{\circ}$ ), so dafs der Anwuchs von $\alpha$ für einen Grad oder $\frac{A \alpha}{\Delta \theta}$ eine constante Gröfse für jede Substanz ist. Die Ausdehnung eines Körpers erfordert also, um vollständig repräsentirt zu seyn, zwei Constanten

$$
\alpha_{F} \text { und } \frac{\Delta a}{\Delta \theta} \text {. }
$$

ist; es scheint mir sogar, dafs die Genauigkeit noch gröfser ist, allein man hat darüber keine Sicherheit. WVenn indefs spätere Versuche eine elwas raschere Verringerung des Indexes, als das Gesetz voraussieht, nachweisen sollten, so wäre es imnuer möglich, alle in dieser Arbeit angeführten Messungen mit Sicherheit zu corrigiren, mittelst der folgenden numerischen Elemente zweier über die Ausdehnung des Platins gemachter Beobachtungen, die unter denen ausgewählt wurden, bei denen die Barometerveränderungen zu vernachlässigen waren und die Dicke der luftschicht $10^{\mathrm{mm}}, 36$ betrug.

Erste Beobachtung.

$t=13^{\circ}, 965 ; t^{\prime}=31^{\circ}, 60 ;$ Verschobene Fransen: $4^{\text {fr }}, 86$

Berichtig. wegen der Luft nach dem Gesetz: $+0^{\mathrm{fr}}, \mathbf{5 6 1}$

$a \vartheta=22^{\circ} 78=0,00000873$.

Zweite Beobacblung.

$t=30^{\circ}, 425 ; t^{\prime}=75^{\circ}, 906$; verschobene Fransen: $13^{\mathrm{fr}}, 035$.

Berichtig. wegen der luft wach $d$. Gesela: $+1^{\mathrm{fr}, 218}$

$u_{y}=53^{\circ} \mathrm{J}=0,00000894$. 
Um diese beiden Constanten mit möglichster Genauigkeit für den platinernen Dreifufs zu bestimmen, machte ich drei Reihep vou Messungen, bei welchen jeder die mittleren Grade etwas verschieden waren.

Die erste Reihe von 9 Beobachtungen gab als Mittel

$$
a=0,00000872665 \quad \vartheta=24^{0}, 9244
$$

Die zweite von 8 Beobachtungen

$$
\alpha=0,0000088592 \quad \vartheta=43^{0}, 4137
$$

Die dritte Reibe von 4 Beobachtungen

$$
\alpha=1,0000059673 \quad \vartheta=56^{0}, 6375
$$

Aus diesen drei Reihen ergeben sich die folgenden definitiven Werthe für die heiden Constanten der linearen Ausdehnung des platinernen Dreifulses ').

$$
\alpha_{s=40}=0,00000883847 ; \frac{A \alpha}{A \theta}=0,7588,
$$

Gebraucht man häufig diesen Coëfficienten für mittlere Grade 9, die bei jedem Versuche variiren, so ist es zur Erleichterung der Rechnungen zweckmäfsig im Voraus eine

1) Die Anwendung aweier Constanteo ist in ler Praxis sehr bequem aur Berechnung aller Ausdehnungsphänomene, wenu man die Veränderung des Coëfficienten für verschiedene Temperaturen in Rechnung zieh. Das. Wesentlichste ist: dals man den VVerth des Coëfficienten nimmt, der dem mittleren Grad zwischen den betrachteten Temperatar-Extremen zukommt. Zu dem Eude muls man dem Coëfficienten $\alpha_{\mathbf{S}=40}$ so oft den Werth $\frac{A \alpha}{\Delta \theta}$ hinzufügen oder von ihn abzieheu, als es Grade giebt von dem betrachteten mittleren Grad bis $z u 40^{\circ}$, sowoll daräber als darunter. Man hat alsdann den mituleren Coëfficienten, welchen wan mittelst der Formel

$$
t_{t^{\prime}}=l_{t}\left[1+\alpha\left(t^{\prime}-t\right)\right]
$$

anwendet.

Um die der cubischen Ausdehnung entsprechenden Werthe zu erhalien, braucht man nur die beiden Constanten mit 3 zu multipliciren.

Ueberdiefs kann man bemerken, dafs wenn man die Ausdehnnng durch die zweigliedrige Formel $l_{t}=l_{0}\left(1+a t+b t^{2}\right)$ ausdrücken will, man leicht die beiden Coëfficienten $a$ und $b$ mittelst der beiden vorhergeheaden belommt, denn man hat:

$$
a=a_{P=0} \text { und } 2 b=\frac{\Delta \alpha}{\Delta \theta} .
$$


Tafel zu entwerfen, welche die Werthe von $\alpha$ von Grad zu Grad zwischen den Gränzen des Temperatur-Umfangs der Beobachtungen angiebt.

Um zu sehen, wie diese 21 partiellen Beobachtungen, die zur Berechnung der beiden Constanten des Platins dienten, übereinstimmen würden, construirte ich eine Curve mit den Werthen von $\vartheta$ als Abscissen und denen von $\alpha$ als Ordinaten. Diese Curve ergab sich nahezu als eine gerade Linie. Zog ich dann die gerade Linie, die aus der Gesammtheit der Beobachtungen für die beiden Constanten hervorging, so liefs sich erkennen, wie stark die partiellen Beobachtungen dies- und jenseits von der die Mittelwerthe repräsentirenden geraden Linie abwichen. Dieser Vergleich zeigte, dafs die Uebereinstimmung der Beobachtungen eine sehr genügende war; und überdiels erlaubte er den Grad der Genauigkeit dieser Methode deutlich einzusehen und abzuschätzen. Die gröfsten Abweichungen entsprachen in gemessenen Längen nämlich: $\pm \frac{1}{40}$ von $\lambda_{D}$ oder beinahe $\frac{1^{\mathrm{mm}}}{65000}$, und die mittleren $\mathbf{A b}$ weichungen nur der Hälfte dieser Länge, d. h. ungefähr $\frac{1}{130000}$ Millimeter.

Nachdem die Ausdehnung des Platin-Dreifulses bestimmt worden, geschieht das Studium der Ausdehnung eines Krystalls oder sonstigen Körpers mit Leichtigkeit bei geringer Dicke von nur einigen Millimetern und mit Hülfe polirter Flächen von ebenfalls sebr geringer Gröfse, denn die Fransen oder Ringe, die man beobachtet, lassen sich durch Fernröhre mit passenden Ocularen vergröfsern.

Ist der Krystall von der Art, dafs er sich nicht recht poliren läfst, so legt man auf seine Oberfläche, auf drei beliebig angebrachten Vorsprüngen ruhend, einen sehr kleinen Spiegel von demselben Platin, aus welchem der Dreiful's besteht, die polirte Fläche gegen die Glasplatte gewandt. Die Fransen bilden sich dann mit Leichtigkeit und die Ausdehnung des Körpers milst sich ebenso gut, wie wenn er allein ohne den Spiegel wäre. Es ist nämlich 
klar, dafs in diesem Fall die Ausdehnung des Spiegels genau compensirt wird durch die Ausdehnung der Schranben, welchen man genöthigt ist ein Verlängerung zu geben genau gleich der Dicke des Spiegels.

Es ist noch hinzuzufügen, dafs man, um dem. Krystall eine vollkommne Unbeweglichkeit $\mathrm{zu}$ geben, veranlafst wurde, ihn auf den Teller des Dreifufses mittelst dreier kleiner stumpfer Spitzen ruhen zu lassen.

Der kleine Raum endlich, worin sich die Ringe bilden und welcher bei den Rechnungen zu einer leichten Correction Anlafs giebt, wird insgemein von derselben Dicke $\varepsilon=0^{\mathrm{mm}}, 02$ genommen, gemessen mittelst $\mathrm{der}$ Theilung auf den Köpfen der Schrauben des Dreifufses.

Die Dicke $e$ der Substanz mufs in allen Fällen zuvörderst mit einem sicheren Sphärometer gemessen worden seyn.

Für jede Substanz machte ich wenigstens drei vollsiăndige Beobachtungen, $d$. $h$. jede 10 Bestinumungen der Lage der Fransen enthaltend, immer bei vollkommen stationären Temperaturen. Es schien zweckmälsiger zu seyn mit der höchsten Temperatur, etwa $\mathbf{7 0}^{\circ}$, anzufangen, dann zu einer weniger hohen, etwa $45^{\circ}$, überzugehen, und endlich auf die gewöhnliche Temperatur zwischen $10^{\circ}$ und $20^{\circ}$ herabzusteigen.

Der Ausdehnungscoëfficient $\alpha$ s der Substanz entsprechend einem mittleren Grad $\vartheta$, berechnet sich mittelst der folgenden Formel:

worin :

$$
\alpha_{\Im}=\frac{\frac{1}{2} f \lambda+\alpha_{S}^{\prime}\left[(e+\varepsilon)\left(t^{\prime}+t\right)\right]}{e\left(t^{\prime}-t\right)}
$$

$\lambda \quad$ Wellenlänge des gelben Strahls $=0^{\mathrm{mm}}, 0005888$; $t$ ' und $t$ höchste und niedrigste Temperatur;

$e$ Dicke der Substanz in Millimetern;

$\varepsilon$ Dicke der Luftschicht, in der sicb die Fransen bilden;

$\alpha^{\prime}$ s Ausdehnungscoëfficient des Platins für den mittle$\operatorname{ren} \operatorname{Grad} \frac{1}{2}\left(t^{\prime}+t\right)$; 
$f$ Anzabl der verschobenen Fransen während die Temperatur von $t$ auf $t^{\prime}$ steigt.

Es ist wichtig zu bemerken, dafs diese letztere Gröfse $f$ das Zeichen + oder - bekommen mufs, je nachdem die Substanz eine gröfsere oder geringere Ausdehuung als die des Platins besitzt; diefs erkennt man sogleich an dem Sinn der Fransenbewegung, die im ersten Fall vom Mittelpunkt der. Ringe zu deren Umfang geht und in letztern Fall umgekehrt (die polirten Flächen inmer schwach convex vorausgesetzt $\left.{ }^{\mathbf{l}}\right)$.

Die drei Beobachtungen liefern somit zwei Werthe von $\alpha$ entsprechend zweien Werthen von $\vartheta$, die um 20 bis 30 Grad von einauder stehen. Man berechnet alsdann die Constante $\frac{\Delta a}{\Delta \theta}=\frac{a-\alpha^{\prime}}{\vartheta-g^{\prime}}$ und mittelst dieser leitet man aus einem der Werthe von $a$ die zweite Constante $\alpha_{g}=40$ $a b, d$. h. den Werth des gesuchten Coëfficienten für einen Grad, der auf dem Punkt $40^{\circ}$ der Thermometerscale liegt.

Ich will nun die Resultate der Versuche mittheilen, die an einer gewissen Anzahl von Körpern, meistens isolirt und wobl hestimmten Krystallen, besonders zur Gruppe der Metalloxyde gehörig, angestellt wurden.

Ein kundiger Mineraloge, Hr. Des Cloi zeaux, hat die Güte gehabt, mich bei der Auswahl und der Bestimmung der Krystalle zu unterstützen, was sehr viel zum Erfolg dieser Untersuchungen beigetragen hat. Ueberdiefs verdanke ich mehrere kostbare Mineralprohen der Gefälligk eit des Hrn. D a mour. Aber besonders zum Dank verpflichtet fühle ich mich allen denen unserer gelehrten Collegen, die

1) Polirte Flächen von kleinen Dimensionen sind in der Regel etwas convex, wenn sie auf gewöhnliche VVeise geschliffen werden. Um einen Irrthum hinsichtlich des Zeichens der Grölse $\boldsymbol{f}$ zu vermeiden, ist es indefs sicheser, diese Convexität durch eine stehr einfache Probe zu ermitteln. Dazu genügt es, bevor man den Dreifufs mit dem darauf liegenden Krystall in den Apparat stellt, die Ringe mit blofsem Auge unter rasch vergrölserten Schiefen zu betrachten. Sipd es die Ringe convexer Flächen, so fliehen sie vom Centrun, während sie sich demselben nähern, wenu die Flächen concav sind. 
mich sowohl oft durch ihren Rath unterstiitzten als mir auch freien Zugang zu unseren grofsen öffentlichen Sammlungen in der École des Mines, im Museum und im Collége de France verstatteten.

Spiegelglas von Saint-Gobain. - Aus einem sehr reinen Stück wurde ein rechtcckiges Parallelepipedon von $10^{\mathrm{mm}}, 057$ Dicke geschnitten; es hatte eine Dichte $=2,514$ und für gelbes Licht einen Brechungsindex $=$ 1,528.

Die cubische Ausdehnung, aus der linearen dureh Multiplication mit 3 hergeleitet, fand sich für $\vartheta=40^{\circ}$

$$
\alpha_{\Im=40}^{\mathrm{cub} .}=0,000012331 ; \frac{A \alpha}{A \vartheta}=4,74 \text {. }
$$

Diamant (dem regulären oder cubischen System angehörig). - Ein sehr klares gelbes, als Brillant geschliffenes Exemplar von 1 grm,94 Gewicht, dem Museum gehörig.

In einer früheren Mittheilung ${ }^{1}$ ) gab ich den sebr schwachen Werth der Ausdehnung des Diatnanten und dessen rasche Abnahme mit der Temperatur, wonach sich für diese Substanz das Daseyn eines Dichtigkeitsmaximums gegen $-40^{\circ} \mathrm{C}$. als sehr wahrscheinlich ergab. Die Administration des Museums war so gut, mir den Stein, an welchem diese Bestimınungen gemacht waren, nochmals anzuvertrauen. Ich konnte ihn nun mit viel gröfserer Genauigkeit untersuchen und erhielt Resultate, welche, was die sehr schwache Ausdehnung der Substanz betrifft, zwar wenig von den früheren abwichen, aber sicherer als jene sind. Die Dicke des Krystalls betrug $9^{\text {ram }}, 625$.

$$
\alpha_{\mathrm{g}=40}^{\text {cub. }}=0,00000354 ; \frac{A \alpha}{\Delta \vartheta}=4,32 \text {. }
$$

Der Punkt der nullgleichen Ausdehnung, d. h. das Maximum der Dichte läge bei $-42^{\circ}, 3 \mathrm{C}$.

Kupferoxydul (dem regulären System angehörig). Bei den früheren Versuchen beobachtete ich an sehr reinen Krystallen von Chessy eine noch schwächere und noch rascher abnehmende Ausdehnung als beim Diamant, so daf's dis Daseyn eines Dichtigkeitsmaximums bei dem Kupfer-

1) Co:npt. rend. T. LX, p. 1161, (Diese Anı. Bd. CXXVI, S. 611). 
oxydul noch wahrscheinlicher war, und dasselbe in der Nähe von $0^{0}$ liegen mufste d. h. wenig entfernt von der Temperatur, auf welche es die Beobachtungen versetzt hatten. Das grofsé Interesse, welches diese Substanz darbot, veranlafste mich, ibre Ausdehnung abermals zu bestimmen, um den Werth der Constanten noch genauer festzusetzen. Sechszehn neue Bestimmungen an verschiedenen uud sehr reinen Exemplareu, bestehend aus isolirten und aus verworren verwachsenen Krystallen, führten zu folgenden definitiven Werthen:

Die Dicken der Krystalle waren $9^{\text {inm }}, 836 ; 12^{\text {mm }}, 999$ und $10^{\mathrm{mm}}, 644$.

$$
x_{\mathrm{g}=40}^{\mathrm{cub} .}=0,00000279 ; \frac{\Delta a}{\Delta \vartheta}=6,30 .
$$

Die Temperatur, bei welcher dieser Körper ein Maximum der Dichte zu besitzen scheint, läge gegen $-4^{0}, 3 \mathrm{C}$.

Smaragd (Beryll von Sibirien; Doppelsilicat von Thonerde und Beryllerde; dem hexagonalen System angehörig). - Die Versuche wurden an mehreren verschiedenen Krystallen gemacht, die meisten Messungen aber an einem sehr reinen Aquamarin von der École des Mines, den ich Hrn. Daubrée verdanke.

Da diese Substanz zu einem um eine Axe symmetrischen Systeme gehört, so hat man zwei Ausdehuungen zu bestimmen, eine nach der Krystallaxe, die mit der optischen Axe und einer der Elasticitätsaxen zusammenfällt, und die andere nach irgend einer auf dieser Axe winkelrechten Richtung.

1. Nach der Axe, Dicke des Krystalls: $10^{\mathrm{mm}}, 748$ und 14 $4^{\mathrm{mm}}, \mathbf{5 6 4}$. - In dieser Richtung beobachtet man eine negative Ausdehnung, d. h. eine recht deutliche Zusammenziehung, deren Coëfficient im absoluten Werth abnimmt mit der Temperatur, nach demselben regelmäfsigen Gesetz wie die positiven Coëfficienten. Die beiden Constanten sind alsdann:

$$
\alpha_{s=40}^{\operatorname{lin} .}=-0,00000106 ; \frac{d a}{\Delta \vartheta}=1,14 .
$$


2. Winkelrecht zur Axe, Dicke des Krystalls: $9^{\mathrm{mm}}, \mathbf{5 9 5}$ und $5^{\text {mm }}, 907$. - In dieser Richtung ist die Ausdehnung positiv, aber sehr schwach

$$
\alpha_{2=40}^{\prime \text { lin. }}=+0,00000137 ; \frac{\Delta \alpha^{\prime}}{\Delta \vartheta}=1,33 \text {. }
$$

Um die cubische Ausdehnung zu erbalten, mufs man die Summe nehmen

$$
2 \alpha^{\prime}+\alpha=\alpha^{\mathrm{cub}} ; 2 \Delta \alpha^{\prime}+\Delta \alpha=\Delta \alpha^{\mathrm{cub}} \text {. }
$$

Somit hat man für die Constanten der cubischen Ausdehnung des Sinaragds (Berylls) folgende Werthe:

$$
\alpha_{S=40}^{\mathrm{cub} .}=+0,00000168 ; \frac{\Delta \alpha}{\Delta \theta}=3,80 \text {. }
$$

Der, gegen die früberen noch schwächere Werth dieser Ausdehnung und seine noch raschere $A$ bnahme bieten eine sehr hervorstechende Analogie mit den entsprechenden Phänomenen beim Diamant und besonders beim Kupferoxydul dar. Denn diese führen für eine wenig unter Null liegende Temperatur zu einem Ausdehnungscoëfficienten $=0$, so dafs der Smaragd (Beryll) wirklich ein Maximum der Dichte bei etwa $-4^{\prime \prime}, 2$ zu haben scheint.

Mehre grüne Smaragde von Columbien und andere von verschiedenen Farben gaben äbnliche Resultate mit kleinen numerischen Abweichungen, die entweder Unreinigkeiten in den Krystallen oder Sprüngen, deren sie oft sehr viele darbieten, zugeschrieben werden können.

Um diese sonderbare Eigenschaft des Berylls wo möglich direct zu erweisen, liefs ich aus einem grofsen sibirischen Krystall eine lange Nadel schneiden, solchergestalt, dafs sie gegen die Axe des Krystalls einen Winkel von $5^{\circ} 4^{\prime}$ machte. Nach dem, was im ersten Theil dieser $\mathbf{A b}$ handlung gesagt worden, ist die Ausdehnung in dieser Richtung die mittlere und zwar rings um die Axe in den Krystallen dieses Systems; denn da zwei der Elasticitätsaxen einander gleich siud, so bleibt ihre Lage rings um die dritte unbestimmt, und das Octaëder der mittleren Ausdehnung kann nach allen möglichen Richtungen rings um die Hauptaxe des Krystalls gelagert werden. Es steht zu erwarten, 
dafs, wenn man diese Nadel in flüssigem Stickstoffoxydul erkaltete, d. h. bis etwa $-78^{\circ} \mathrm{C}$, und sie dann unter einen sebr empfindlichen Fühlhebel (sphéromètre à levier) auf die gewöhuliche Temperatur zurückkommen liefse, es möglich seyn würde, sich direct zu versichern, ob die Substanz bei der Erwärmung von diesen niederen Temperaturen aus sich wirklich zusammenziehe, wie es die neueren Beobachtungen anzunehmen nöthigen.

Quarz (Bergkrystall zum rhomboëdrischen System gehörend). - Die früheren Bestimmungen über den Quarz ') wurden wiederholt, um genauere numerische Werthe zu erhalten

1. Nach der Axe, Dicke $=15^{\mathrm{mm}}, 054$ :

$$
\alpha_{\mathrm{S}=40}^{\text {lin. }}=0,00000781 ; \frac{\Delta \alpha}{A \theta}=1,77 .
$$

2. Winkelrecht zur Axe, Dicke $=15^{\mathrm{mm}, 057}$ :

$$
\alpha_{g=40}^{\operatorname{lin} .}=0,00001419 ; \stackrel{A \alpha}{A \vartheta}=2,38 .
$$

Cubische Ausdehnung des Quarzes:

$$
\alpha_{\mathrm{g}=40}^{\mathrm{cab} .}=0,00003619 ; \frac{A \alpha}{A \theta}=6,53 .
$$

Rutil (Titansäure, System des geraden quadratischen Prismas). - Die Versuche wurden mit einem schönen Krystall von Limoges aus der Sammlung des Hrn. Des Cloizeaux gemacht. Ausdehnung sehr stark.

1. Nach der Axe, Dicke $=12^{\text {min }}, 863$ :

$$
\alpha_{S=40}^{\operatorname{lin} .}=0,00000919 ; \frac{A \alpha}{A g}=2,25 \text {. }
$$

2. Winkelrecht zur Axe, Dicke $=7^{\mathrm{mm}}, 071$ :

$$
\alpha \underset{S=40}{\operatorname{lin} .}=0,00000714 ; \frac{A \alpha}{\vartheta A}=1,10 \text {. }
$$

Cubische Ausdehnung des Rutils:

$$
\alpha_{g=40}^{c u b .}=0,00002374 ; \frac{A a}{A \vartheta}=4,45 \text {. }
$$

Cassiterit (Zinnoxyd, System des geraden quadratischen 1) Compt. rend. 1864, T. LVIII, p. 923, (Ann. Bd. CXXIII, S. 515). 
Prismas). - Krystall aus Sachsen, anscheinend ohne Zwilling, von schwarzer Farbe. Die beiden Ausdehnungen sehr schwach.

1. Nach der Axe, Dicke $=10^{\mathrm{nm}}, 399$ :

$$
\alpha_{\mathrm{S}=40}^{\operatorname{lin} .}=0,00000392 ; \frac{A \alpha}{\Delta \theta}=1,19 .
$$

2. Winkelrecht zur Axe, Dick $\mathrm{e}=11^{\mathrm{mm}}, 302$ :

$$
\alpha_{S=40}^{\operatorname{lin} .}=0,1011000321 ; \frac{\Delta \alpha}{A \vartheta}=0,76 .
$$

Cubische Ausdehnung des Zinnoxyds:

$$
\alpha_{\mathrm{T}=40}^{\text {cub. }}=0,00001034 ; \frac{A \alpha}{A \theta}=2,71 \text {. }
$$

Periclas (Magnesia, zum regulären System gehörig). Die Versuche wurden mit künstlichen, von Hrn. Henri Sainte-Claire-Deville erhaltenen, verwachsenen Krystallen (Octaëdern) angestellt. Ausdehnung ziemlich stark.

Cubische Ausdehnung der reinen Magnesia:

$$
a_{\xi=40}^{\mathrm{cub} .}=0,00003129 ; \frac{\Delta \alpha}{A \vartheta}=8,01 .
$$

Spartalit (Zinkoxyd, hexagonales System). - Natürliche Krystalle aus den Vereinigten Staaten, von rother Farbe, aus der Sainmlung des Hrn. Des Cloizeaux. Ausdehnung schwach.

1. Nach der Axe, Dicke $=7^{\mathrm{nm}}, \mathbf{5 5 5}$ :

$$
\alpha_{\mathrm{q}=40}^{\operatorname{lin} .}=0,00000316 ; \frac{\Delta \alpha}{\Delta \theta}=1,86 \text {. }
$$

2. Winkelrecht zur $A x e$, Dicke $=9^{\mathrm{mm}}, 118$ :

$$
\alpha_{q=40}^{\operatorname{lin} .}=0,00000539 ; \frac{A \alpha}{\Delta q}=1,23 \text {. }
$$

Cubische Ausdehnung des Zinkoxyds:

$$
\alpha_{\text {श }=40}^{\text {cub. }}=0,00001394 ; \frac{\Delta a}{\Delta \vartheta}=4,32 \text {. }
$$

Korund (Reine Thonerde, rhomboëdrisches System). Ein schönes Exemplar, aus der École des Mines, welches ich Hrn. Daubrée verdanke, wurde nach den beiden, für diese Versuche nöthigen Richtungen geschnitten. Es war 
ein bläulicher, etwas milchiger Korund aus Indien. Ausdebnung schwach.

1. Nach der Axe, Dicke $=10^{\mathrm{mm}}, 027$ :

$$
\alpha_{\mathrm{q}=40}^{\operatorname{lin} .}=0,00000619 ; \frac{d \alpha}{d \theta}=2,05 .
$$

2. Winkelrecht zur Axe, Dicke $=7^{\mathrm{mm}}, 265$ :

$$
\alpha_{g=40}^{\operatorname{lin} .}=0,00000533 ; \frac{\Delta \alpha}{\Delta \vartheta}=2,25 \text {. }
$$

Cubische Ausdehnung des Korunds:

$$
\alpha_{s=40}^{\mathrm{cub} .}=0,00001705 ; \frac{\Delta a}{\Delta \theta}=6,55 \text {. }
$$

Eisenglanz (Eisenoxyd, rhomboëdrisches System) Krystall von Elba, äufserlich sehr nett, in welchem aber der Schnitt einige Unvollkommenheiten aufdeckte. Die Messungen müssen daher an einem anderen Krystall wiederholt werden. Die beiden Ausdehnungen sehr wenig verschieden und von mittlerer Grölse.

1. Nach der Axe, Dicke $=9^{\text {mm }}, 581$ :

$$
\alpha_{\mathrm{S}=40}^{\text {lin. }}=0,00000829 ; \frac{\Delta \alpha}{\Delta \theta}=1,19 .
$$

2. Winkelrecht zur Axe, Dicke $=9^{\text {min }}, 146$ :

$$
\alpha_{S=40}^{\operatorname{liu} .}=0,00000836 ; \frac{A a}{A \theta}=2,62 \text {. }
$$

Cubische Ausdehnung des Eisenglanzes:

$$
\alpha_{\substack{c u b . \\ c=40}}^{c u, 00002501 ;} \frac{\Delta \alpha}{\Delta \theta}=6,43 \text {. }
$$

Senarmontit (Antimonige Säure, regelmäfsiges System). - Sehr nettes Octaëder von Constantine, etwas milchig, aus der École des Mines. Ausdehnung sehr beträchtlich.

$$
\alpha_{\substack{\mathrm{cob} . \\ \mathrm{cot}}}^{\mathrm{c}}=0,00005889 ; \frac{\Delta \alpha}{A \vartheta}=1,71 .
$$

Arsenige Säure (Octaëdrische Krystalle des regulären Systems). - Sehr schőne künstliche Krystalle aus dem Laboratorium der École normale von Hrn. H. St.-Claire Deville erhalten. Ausdehnung ungewöholich, stärker als alle vorhergehenden 


$$
\alpha_{\mathrm{P}=40}^{\mathrm{cub} .}=0,00012378 ; \frac{\Delta a}{\Delta \theta}=20,37 .
$$

Spinelle (Octaëdrische Krystalle des regulären Systems). - Schliefslich gebe ich noch die Ausdehnung ron vier Spinell-Arten (Thonerde und Magnesia oder Zinkoxyd und deren Isomorphe), deren Werthe einander merkwürdig nabe koinmen

1. Rubin, von Ceylon, ans dem Museum:

$$
\alpha_{\mathrm{g}=40}^{\text {cub. }}=0,00001787 ; \frac{A \alpha}{A \theta}=7,29 \text {. }
$$

2. Pleonast, Eisenoxyd ron Warwick:

$$
a_{q=40}^{c a b .}=0,00001180.5 ; \frac{A a}{d \theta}=5,34
$$

3. Gahnit, Zinkoxyd vou Fablun:

$$
\alpha_{\text {sub. }}^{\text {cub }}=0,00001766 ; \frac{\Delta \alpha}{\Delta 9}=5,19 .
$$

4. Kreittonit, von Silberberg:

$$
\alpha_{\mathrm{P}-40}^{\text {cub. }}=0,001101750 ; \frac{A \alpha}{A \vartheta}=5,31 .
$$

Die Fortselzung dieser Untersuchungen wird Gegenstaud einer zweiten Abhandlung seyn, in welcher alle numerischen Resultate miteinauder verglichen und in ihrer Gesammtheit discutirt werden sollen.

\section{Ueber den Lullin'schen V'ersuch und die \\ Lichtenberg'schen Figuren; con Dr. A. von W'altenhofen.}

Die elektrischen Erscheinungen, welche zuerst in Jahre $1840 \mathrm{zu}$ Seghill an einem Dampfkessel beobachtet uud bald darauf von Armstrong und Pattinson und vornehmlich von Faraday gründlich erforscht worden sind, haben zu höchst wichtigen Fortschritten im Gebiete der 